\title{
Challenges and updates in the management of eosinophilic esophagitis
}

\author{
Tsvetelina Velikova \\ Department of Clinical Immunology, University Hospital Lozenetz, Sofia, Bulgaria
}

Gastroenterology Rev 2020; 15 (1): 27-33

DOI: https://doi.org/10.5114/pg.2019.84476

Key words: esophagitis, eosinophilia, diet, corticosteroids, esophageal dilatation.

Address for correspondence: Tsvetelina Velikova MD, PhD, Department of Clinical Immunology, University Hospital Lozenetz, Sofia, Bulgaria, phone: +3 59883306949 , e-mail: tsvelikova@medfac.mu-sofia.bg

\begin{abstract}
Eosinophilic esophagitis (EE) is a chronic T helper cell-2 mediated inflammatory condition and is considered as a primary cause of dysphagia and foregut symptoms. There are many challenges regarding the treatment options of EE. Different therapeutic approaches are best at meeting different endpoints. There are unresolved questions such as whether the goal for treatment should be to control esophageal eosinophilia and inflammation or to achieve symptomatic improvement. Still, proton pump inhibitors are used in differential diagnosis, along with their anti-inflammatory and anti-acid properties. Conducted trials continue to recommend the use of topical corticosteroids and empiric food elimination diets as first-line therapeutic modalities. The growing knowledge on the pathogenesis of EE has allowed further progression of encouraging targeted biologic therapies. However, the guidelines for EE management should be updated accordingly in the coming years, including fast evolving data on non-invasive diagnostic strategies, new treatment modalities, and the long-term prognosis of the disease.
\end{abstract}

\section{Introduction}

Eosinophilic esophagitis (EE) is a chronic Th2 (T helper cell) mediated inflammatory condition characterized histologically by predominant and marked eosinophilic infiltrate of the esophageal mucosa with subsequent development of fibrosis [1]. Clinically, it is characterized by symptoms related to esophageal dysfunction which progresses chronically and reduces the quality of life, although research has not shown any amplified risk of cancer or mortality [2].

Initially, EE was regarded as very rare and has evolved from sporadic case reports to become a widely recognized cause of esophageal morbidity. It became evident that its incidence has risen rapidly over the last 15 year from 0.35 cases $/ 100000$ people to 9.5 cases/100 000 people in the US [3]. The reasons for this rapid increase are poorly understood, and improved recognition is not the effect of raised awareness [4]. Several population-based studies from Europe (Switzerland, Netherlands, and Denmark) have also provided evidence for a true increase $[3,5,6]$.

Although EE mainly affects persons $20-40$ years old, it can be seen in all age groups. EE has a known male predominance, with a male-to-female ratio approaching $3: 1$ [4]. Around $50-60 \%$ of patients have a personal history of atopic dermatitis prior to being diagnosed with EE. A recent meta-analysis confirmed higher prevalence of EE in adults than in children, 43.4/100 000 vs. $29.5 / 100000$, meaning that one in every 3000 citizens in Westernized areas have EE $[7,8]$. However, the incidence and prevalence of EE are comparable with the values of Crohn's disease [4].

Genetic studies have identified multiple genes possibly contributing to the development of $\mathrm{EE}$, including CCL26 (encoding eotaxin-3) and encoding calpain 14 (CAPN14), thymic stromal lymphopoietin (TSLP), STAT6, etc. [2]. However, a substantial influence is attributed to the environment and epigenetic regulation $[9,10]$.

The pathogenesis of EE results from the complex interaction between genetics, environment, and antigenic stimuli from food or aerosols. The allergen exposure triggers the antigen presentation and differentiation of Th2 cells secreting interleukin (IL)-4 IL-5 and IL-13. These cytokines further enhance the production of other mediators such as CCL26/eotaxin-3 and upregulation of periostin in epithelial cells and fibroblasts. Calpain, desmoglein, and filaggrin along with recruit- 
ment and simulation of eosinophils lead to disruption of the epithelial barrier of the esophagus. Eosinophils also release IL-9 with a possible effect on mast cells. The pathophysiological mechanism also includes basophils and plasma cells rich in IgG4 which are found abundantly in the lamina propria but with unclear significance. Subepithelial fibrosis, mediated by TGF- $\beta$ along with IL-13, contribute to the remodeling of the lamina propria, which is a common complication of the disease [1].

Clinical signs of EE vary substantially among patients, ranging from no prominent symptoms to intermittent dysphagia due to consumption of certain solid foods, to repeated food impaction practically every day. A mild form of EE usually does not disturb patients, which may lead to a delay in diagnosis [11]. Other symptoms associated with EE are heartburn, regurgitation and chest discomfort.

The current consensus criteria defining EE included symptoms of the esophagus and marked eosinophilic inflammation of the esophagus (histologically a peak count of 15 eosinophils per high-power field of esophageal biopsy tissue). Recently, it was documented that the proton pump inhibitor (PPI) responsiveness is not part of the diagnostic criteria but rather an appropriate and effective treatment for some patients [1].

However, despite growing efforts to elucidate its pathogenesis and the increasing number of well-designed clinical trials, the practical management of EE is often challenging because of controversies related to therapeutic endpoints and the need for long term treatments. Here, we conducted a thorough review of the literature, with an emphasis on recent discoveries and updates on management and treatment of EE, as well as on the unresolved problems, focusing mainly on adults with the disease.

\section{Treatment of EE}

Treatment of EE still has some challenges. First, the aim of treatment should be determined, since different therapeutic approaches are best at meeting different endpoints. There are unresolved questions such as whether the goal for treatment should be to control esophageal eosinophilia and inflammation or to achieve a symptomatic improvement. However, histological and clinical remissions differ significantly and patients often exhibit symptoms even after endoscopic remission has been achieved [12]. Usually, histological improvement is used as the primary outcome instead of subjective symptomatic improvement. This discrepancy can be explained by the limited effect of currently available medication on the sub-epithelial fibrosis and remodeling Second, the limitation of the treatments is seen clearly after discontinuation of medications, when EE easily recurs. Thus, appropriate maintenance therapy has yet to be established.

Since the ideal target for pharmacological therapy is not yet established, the search for a suitable target continues. However, it is accepted that symptom relief is not an optimal target due to lack of association with histological remission [13]. Therefore, it has been proposed that the combination of histological remission along with reduction of eosinophil counts $>90 \%$ than baseline or below $6 / \mathrm{hpf}$ could be used as a criterion for EE remission [14].

The established therapeutic approach for the moment is known as the "3D" concept, which stands for diet, dilation, and drugs [15]. Initially, the treatment is chosen based on the severity of the patient's symptoms or endoscopic findings. Complaints of esophageal narrowing or stricture are taken into account when choosing a therapeutic strategy. Control of esophageal inflammation may eliminate the need for mechanical dilation to reduce dysphagia and prevent food impaction. Fortunately, the latter can also be achieved by modifying the patient's dietary or eating behavior [12].

Since EE is similar to allergic airway diseases, drugs used to treat asthma, and acid-suppression agents have been tested as treatments of EE. In the last few years, results from several double-blind controlled randomized clinical trials have aided physicians in optimizing treatment decisions [12]. Nevertheless, the best management of patients with EE could be provided with a team that includes specialists of gastroenterology, allergy, and nutrition.

\section{Dietary therapy}

As a first-line treatment for patients with $E E$, diet modifications are increasingly accepted. They possess high efficacy, low cost, and a good safety profile. Furthermore, diet therapy can directly address the underlying allergic mechanism. Dietary treatment is useful in identifying a limited number of food antigens that could trigger inflammation [16]. However, a significant drawback is the multiple endoscopic examinations individual patients undergo in an attempt to identify the triggering foods. Less invasive testing with a swallowed sponge administered at the bedside without anesthesia is currently being studied [11].

Various dietary treatment modalities have been proposed and can be divided into three main categories: the elemental diet, empiric elimination diets, and elimination diet guided by allergy testing. They have been attempted primarily in infants and more recently, in adults with EE [16]. 
The high efficacy of the elemental diet in inducing clinical and histologic remission of patients with $E E$ was first shown in 1995. The proposed diet is based on using an amino acid-based formula, lacking proteins or potential dietary antigens, which requires a feeding tube or has an unpleasant flavor [8]. Nevertheless, its extreme cost and poor tolerability, as well as the negative impact on quality of life linked to the avoidance of all table foods, make it unsustainable for long-term use.

Related to the elemental diet is the six-food elimination diet (SFED), which excludes empirically the top six food groups most commonly associated with food allergy (cow's milk, wheat, egg, soy, peanut/tree nut, and fish/seafood) and a subsequent reintroduction phase involving performing a number of endoscopies with histologic examination to monitor the response [17]. The top four causative foods for EE, identified as cow's milk, wheat, egg, and soy/legumes, is the basis for establishing the four-food elimination diet (FFED) [18]. The level of dietary restriction in SFED may be unnecessary in many cases leading to patient refusal or low compliance with this diet. The benefits of FFED include fewer restrictions, better acceptance by patients, and potentially a shorter study time with fewer endoscopies needed to identify food triggers [8].

Also a step-up elimination diet, first excluding the top two foods (milk and wheat), then the top four, then the top six has been proposed in an abstract. Compared to other elimination diets, this step-up approach enables reduction of diagnostic time by $35 \%$, prevention of unnecessary dietary restriction, and reduction in the number of endoscopic procedures by $25 \%$ [19]. Thus, by identifying and determining the food triggers responsible for inducing $E E$ in an individual patient, a maintenance avoidance diet showed efficacy in clinical and histologic remission for years [8].

To resolve the drawbacks of the elimination diets, allergy testing based elimination diet therapy has been introduced. Despite demonstrating high efficacy for this targeted elimination therapy, the capacity to predict the causative allergens is relatively low using the currently available allergic tests (skin prick test, patch test, and serum food antigen-specific lgE testing). The reasons for this are not fully clear but may be partly because of the involvement of non-lgE-mediated hypersensitivity and delayed-type reactions [8].

Thus, diet treatment may be a useful therapeutic option that even allows the decrease or cessation of medications, especially in patients who require longterm use of steroid therapy [20]. Diet therapy in EE, however, has some concerns, such as the increased cost of food, poor compliance, and nutritional deficiencies due to food elimination.

\section{Pharmacologic therapy}

The latest guideline for therapy management of EE was published in 2017 [21, 22]. These guidelines emphasize the exclusion of a condition called PPI-responsive esophageal eosinophilia (PPI-REE) by a 2-month course of PPI followed by endoscopic investigations with biopsies. The advantages of medical treatment with PPIs include effectiveness with few risks at low costs [21]. Recommendations on treatment endpoints continue to be "conditional" or based on the relatively low quality of evidence or lack of supporting data [8]. However, for the first-line treatment for EE inflammation it is recommended to administer topical steroids (specifically budesonide and fluticasone) along with dietary elimination. Corticosteroids through their ability to inhibit differentiation and activation of eosinophils through suppression of their prerequisite cytokines have been confirmed effective in managing EE [23]. Esophageal dilatation is suggested in symptomatic patients when they show evidence of strictures.

\section{Acid suppression}

As mentioned above, PPIs may play a role in the diagnostic evaluation of patients with suspected EE and the care of diagnosed patients. The PPIs are the first line treatment if patients fail or do not tolerate dietary therapy. The PPIs are initially given for 8 weeks and depending on the response they are continued or upper endoscopy is pursued [24]. A lack of response to PPIs is currently the only criterion available to rule out gastroesophageal reflux disease (GERD) as a cause of esophageal eosinophilia while having in mind that patients with well-established EE can also have symptomatic GERD that is responsive to PPI and contributes to the development of EE [25]. In vitro studies showed that independently of their effect on acid secretion, PPIs also decrease cytokine secretion from the esophageal epithelium. Thus, the anti-inflammatory properties of PPIs were suggested [25]. Studies on patients with PPIREE showed that their clinical, histologic, and genetic characteristics are similar to patients with EE, which supports the hypothesis that PPI-REE might be a subtype of EE [26]. Nevertheless, as mentioned above, PPIs may distinguish both disease entities [27].

\section{Topical glucocorticoids}

Topical corticosteroids are effective in children and adults and are considered the other first-line treatment option for EE. Furthermore, oral viscous budesonide has been shown to have a greater mucosal contact time than the nebulized solution [8].

Overall, topical corticosteroids as compared with placebo were effective in inducing complete histologic 
remission in $57.8 \%$ to $82.1 \%$ of $\mathrm{EE}$ cases but were not associated with a significant improvement in clinical symptoms [28]. These observations may be explained in part with the high placebo response rates. Nevertheless, topical steroids were well tolerated among patients in all studies. However, esophageal candidiasis was observed in $4 \%$ to $26 \%$ of treated EE patients, although they responded well to oral nystatin treatment. Additionally, no significant adrenal suppression was reported during topical corticosteroid use [28]. Along with other effects, use of topical glucocorticoids may diminish the incidence of subsequent food impactions [29].

\section{Fluticasone propionate}

Fluticasone propionate as a nebulizer has been proposed mainly as an initial regimen. However, it was recently displaced by a viscose type budesonide due to its reliable and uniform delivery to the whole esophageal mucosa in both adults and children [30]. The recommended dose for topical fluticasone propionate steroids is $440-880 \mu \mathrm{g}$ for 8 weeks in adults [21]. It is important for patients with EE to adhere strictly to the instructions for using inhaling corticosteroids to deliver the right dose of the drug, as well as to avoid oral candidiasis [12].

\section{Budesonide}

Budesonide has been evaluated in randomized trials in the treatment of EE. This was confirmed by the first systematic review and meta-analysis conducted by Rawla et al. in 2018, comprising both randomized and non-randomized studies, which included 12 studies and a total of 555 participants [31]. This study showed that topical budesonide exerts substantial effects at all treatment endpoints [31]. This meta-analysis has been used as a guideline for the appropriate usage of budesonide in the treatment of EE. A significant effect of budesonide in establishing histological remission and improvement of clinical symptoms was observed. Moreover, the eosinophilic count has been reduced noticeably during budesonide treatment. However, an increased risk of candidiasis is also documented for patients with EE on budesonide, although more research is necessary to elucidate these results [31]. A significant effect of budesonide was observed at a dose of 1-2 mg twice daily in adults and $0.25-0.5 \mathrm{mg}$ twice a day in children. One study revealed that oral viscous budesonide has more extensive contact with esophageal mucosa and covers a larger extent of the esophagus than nebulized budesonide [32].

\section{Ciclesonide}

Ciclesonide is a topical steroid of proven benefit in the treatment of allergic diseases (i.e., asthma, al- lergic rhinitis, and allergic conjunctivitis), and thus is proposed for EE treatment as well [33]. An insufficient number of studies have demonstrated that EE patients on topical ciclesonide experienced both a clinical and histologic improvement, as well as significantly decreased eosinophil numbers in proximal biopsy specimens. Ciclesonide might offer benefit in treating children with EE as well; however, because of the preliminary nature of the studies, caution with ciclesonide use should be applied. Nonetheless, further clinical and mechanistic studies of ciclesonide in the treatment of EE are needed [34].

\section{Maintenance therapy}

Having in mind the progressive fibrosis accompanying $E E$, maintenance treatment is recommended, particularly in patients with severe symptoms and a history of high-grade strictures, to prevent complications [8]. Long-term maintenance therapy for EE is a controversial topic. EE is not a premalignant disease and does not reduce the life span of the patients. The evidence to date also indicates that a period of long remission (spontaneous or dilation-induced) may occur without the necessity for nutritional alterations or medication use [35]. However, being a chronic disease, EE is characterized by recurrence if treatment is stopped. Due to ongoing inflammation, symptoms recur; quality of life declines and complications such as strictures may develop. A few studies proposed diet or topical glucocorticoid therapy in EE as long-term options for maintaining remission [4]. Thus, maintenance therapy is indicated whenever evidence of chronic remodeling (i.e., symptomatic or objective progression of strictures, a small caliber esophagus), recurrent food impactions, severe symptoms, or rapid return of symptoms while not receiving therapy is observed [27]. Other candidates for long-term maintenance of pharmacologic therapy for EE are the following: co-morbid conditions that increase the risk of endoscopy and dilation, prior spontaneous or dilation-induced perforation, and travel to regions where food impaction causes higher risk [4].

Although corticosteroids' withdrawal leads to relapse at a high rate, Straumann et al. reported the efficacy of low-dose budesonide $(0.25 \mathrm{mg})$ as maintenance therapy in adult EE patients [4, 12]. However, dose reduction should be considered when targeting clinical remission [12].

\section{Topical versus systemic glucocorticoids}

Adverse effects seem to be less often associated with topical therapy than with systemic steroid treatment. Mild or asymptomatic oral candidiasis can arise 
in up to $10 \%$ of patients [36]. It was estimated that up to $10 \%$ of children treated with topical steroids for more than 6 months showed adrenal insufficiency, evaluated by an adrenocorticotropic hormone stimulation test [37]. Even though such cases have not been described in adults, attention should be paid for long-term users of topical steroids [12].

Recent genomic studies may help recognize those EE patients who will be more or less likely to have a response to steroid therapy [38]. Because swallowed topical steroids go through the first-pass metabolism, effects such as adrenal axis suppression, bone demineralization, and diminished growth appear to be uncommon. It was shown that systemic glucocorticoids for EE treatment exert the same therapeutic results but sustain a greater risk of side effects than the use of topical glucocorticoids [27].

\section{Esophageal dilation}

Even though some anti-inflammatory agents may improve fibrosis at the mucosal level, in most EE patients esophageal dilatation is vital to providing instant and long-lasting relief of dysphagia. Although esophageal tear is associated with the need for hospitalization, post-procedural retrosternal pain (in $74 \%$ of cases), the risk of perforation, etc., the rates of complications were reported as $<1 \%$. Thus, it is recommended to start initially with drug or dietary therapy for EE patients whenever possible [39]. If symptomatic EE patients possess esophageal strictures or narrowing despite medical therapy, they require dilation. Since esophageal remodeling develops progressively during long-term and persistent eosinophilic inflammation, it is uncommon in children [40]. Esophageal dilatation is reserved for patients who fail conservative management. But sometimes it may be used as initial therapy for patients who have high-grade strictures [14].

Three categories of procedures have principally been used: the simple bougie, the wire-guided bougie, and through-the-scope (TTS) balloon dilation [41]. The TTS methods showed the potential to prolong the esophageal lumen further than the bougie method, while no substantial difference in complications was observed. It is crucial for the endoscopists to gradually and slowly dilate since chest pain or mucosal tears can happen due to esophageal mucosal delicacy. In patients with $\mathrm{EE}$, the risk factors for dilation-related adverse events are younger age, numerous dilations, strictures in the upper portion of the esophagus, and the failure to pass through the strictures with the endoscope [42]. The majority of patients exhibit symptomatic improvement following dilation; however, its long-term effects appear to be disappointing. Moreover, about half of the patients experienced repetitive dilatations, particularly within the first year. The presence or absence of concomitant anti-eosinophilic medication does not influence the efficacy of endoscopic dilatation [12].

\section{Experimental treatments}

Although most patients with EE respond with histologic reduction of eosinophilia and symptomatic improvement due to topical corticosteroids or dietary elimination, a subset of patients remain non-responders to standard therapy. Experimental treatments have been explored, including antihistamines, a mast cell stabilizer (cromolyn sodium), and leukotriene receptor antagonist (montelukast), but benefits for the patients with $\mathrm{EE}$ have been inadequate [39]. Thus, their role in EE treatment is not established yet [43]. A case series evaluating the effect of infliximab, an anti-tumor necrosis factor in EE, did not lead to any symptomatic or histologic improvement [44].

A prostaglandin D2 receptor antagonist, or $\mathrm{CRTH} 2$ antagonist (OC000459), showed only uncertain improvement in esophageal eosinophilia and symptoms in adults with the refractory disease [45]. Regarding biologic therapy of EE, drugs targeting IL-5, IL-13, and IgE have been explored as potential treatments for refractory EE. Despite anti-IL-5 therapies, including mepolizumab and reslizumab, showing a decrease in the eosinophil count in the esophageal mucosa, they did not achieve histologic remission or clinical improvement $[46,47]$. Several trials have investigated the use of an anti-lgE antibody, omalizumab, but no clinical or histologic disease remission was seen in EE patients, probably because this condition is not primarily an IgE-mediated disease [48]. However, further examination is required, but a combination of medications that inhibits mast cells and eosinophils might be sufficient for the management of the refractory disease.

\section{Conclusions}

In the past few years, EE has grown from a comparatively unknown and underdiagnosed condition to a well-defined disease. The intensive studies of the disease revealed many of the pathophysiological features of $E E$, leading to a considerable breakthrough in therapy management. Efforts in managing EE are being devoted to optimizing dietary elimination protocols, and to investigating additional experimental treatment options, such as biologic drugs, for treatment of refractory EE. Despite the controversies regarding managing of EE, the guidelines will need to be updated accordingly in the coming years, including fast evolving data on non-invasive diagnostic strategies, new treatment modalities, and the long-term prognosis of EE. 


\section{Conflict of interest}

The author declares no conflict of interest.

\section{References}

1. Clayton F, Peterson K. Eosinophilic esophagitis: pathophysiology and definition. Gastrointest Endosc Clin N Am 2018; 28: 1-14.

2. O'Shea KM, Aceves SS, Dellon ES, et al. Pathophysiology of eosinophilic esophagitis. Gastroenterology 2018; 154: 333-45.

3. Prasad GA, Alexander JA, Schleck CD, et al. Epidemiology of eosinophilic esophagitis over three decades in Olmsted County, Minnesota. Clin Gastroenterol Hepatol 2009; 7: 1055-61.

4. Straumann A, Katzka DA. Diagnosis and treatment of eosinophilic esophagitis. Gastroenterology 2018; 154: 346-59.

5. van Rhijn BD, Verheij J, Smout AJ, Bredenoord AJ. Rapidly in creasing incidence of eosinophilic esophagitis in a large cohort. Neurogastroenterol Motil 2013; 25: 47-52e45.

6. Hruz P, Straumann A, Bussmann C, et al. Escalating incidence of eosinophilic esophagitis: a 20-year prospective, population-based study in Olten County, Switzerland. J Allergy Clin Immunol 2011; 128: 1349-50.e5.

7. Arias A, Pérez-Martínez I, Tenías JM, Lucendo AJ. Systematic review with meta-analysis: the incidence and prevalence of eosinophilic oesophagitis in children and adults in popula tion-based studies. Aliment Pharmacol Ther 2016; 43: 3-15.

8. Chen JW, Kao JY. Eosinophilic esophagitis: update on management and controversies. BMJ 2017; 359: j4482.

9. Kottyan LC, Davis BP, Sherrill JD, et al. Genome-wide association analysis of eosinophilic esophagitis provides insight into the tissue specificity of this allergic disease. Nat Genet 2014 46: 895-900

10. Lim EJ, Lu TX, Blanchard C, Rothenberg ME. Epigenetic regulation of the IL-13-induced human eotaxin-3 gene by CREB-binding protein-mediated histone 3 acetylation. J Biol Chem 2011; 286: 13193-204.

11. Reed CC, Dellon ES. Eosinophilic esophagitis. Med Clin North Am 2019; 103: 29-42.

12. Abe Y, Sasaki Y, Yagi M, et al. Diagnosis and treatment of eosinophilic esophagitis in clinical practice. Clin J Gastroentero 2017; 10: 87-102.

13. Safroneeva E, Straumann A, Coslovsky M, et al. Symptoms have modest accuracy in detecting endoscopic and histologic remission in adults with eosinophilic esophagitis. Gastroenterology 2016; 150: 581-90.e584.

14. Dellon ES, Gonsalves N, Hirano I, et al. ACG clinical guideline: evidenced based approach to the diagnosis and management of esophageal eosinophilia and eosinophilic esophagitis (EoE). Am J Gastroenterol 2013; 108: 679-92; quiz 693.

15. Singla MB, Moawad FJ. An overview of the diagnosis and management of eosinophilic esophagitis. Clin Transl Gastroentero 2016; 7: e155.

16. Arias A, González-Cervera J, Tenias JM, Lucendo AJ. Efficacy of dietary interventions for inducing histologic remission in patients with eosinophilic esophagitis: a systematic review and meta-analysis. Gastroenterology 2014; 146:1639-48.

17. Lucendo AJ, Arias A, Gonzalez-Cervera J, et al. Empiric 6-food elimination diet induced and maintained prolonged remission in patients with adult eosinophilic esophagitis: a prospective study on the food cause of the disease. J Allergy Clin Immunol 2013; 131: 797-804.

18. Molina-Infante J, Arias A, Barrio J, et al. Four-food group elimination diet for adult eosinophilic esophagitis: a prospective multicenter study. J Allergy Clin Immunol 2014; 134: 10939e1091.

19. Molina-Infante J, Arias A, Alcedo J, et al. Step-up empiric elimination diet for pediatric and adult eosinophilic esophagitis: the 2-4-6 study. J Allergy Clin Immunol 2018; 141: 1365-72.

20. Hirano I. 2015 David Y. Graham Lecture: the first two decades of eosinophilic esophagitis - from acid reflux to food allergy. Am J Gastroenterol 2016; 111: 770-6.

21. Dellon ES, Gonsalves N, Hirano I, et al. ACG clinical guideline: evidenced based approach to the diagnosis and management of esophageal eosinophilia and eosinophilic esophagitis (EoE). Am J Gastroenterol 2013; 108: 679-92.

22. Lucendo AJ, Molina-Infante J, Arias A, et al. Guidelines on eosinophilic esophagitis: evidence-based statements and recommendations for diagnosis and management in children and adults. United European Gastroenterol J 2017; 5: 335-58.

23. González-Cervera J, Lucendo AJ. Eosinophilic esophagitis: an evidence-based approach to therapy. I Investig Allergol Clin Immunol 2016; 26: 8-18.

24. Vazquez-Elizondo G, Ngamruengphong S, Khrisna M, et al. The outcome of patients with oesophageal eosinophilic infiltration after an eight-week trial of a proton pump inhibitor. Aliment Pharmacol Ther 2013; 38: 1312-9.

25. Cheng E, Zhang X, Huo X, et al. Omeprazole blocks eotaxin-3 expression by oesophageal squamous cells from patients with eosinophilic oesophagitis and GORD. Gut 2013; 62: 824-32.

26. Wen T, Dellon ES, Moawad FJ, et al. Transcriptome analysis of proton pump inhibitor-responsive esophageal eosinophilia reveals proton pump inhibitor-reversible allergic inflammation. J Allergy Clin Immunol 2015; 135: 187-97.

27. Furuta GT. Eosinophilic esophagitis: update on clinicopathological manifestations and pathophysiology. Curr Opin Gastroenterol 2011; 27: 383-8.

28. Murali AR, Gupta A, Attar BM, et al. Topical steroids in eosinophilic esophagitis: systematic review and meta-analysis of placebo-controlled randomized clinical trials. J Gastroenterol Hepatol 2016; 31: 1111-9.

29. Kuchen T, Straumann A, Safroneeva E, et al. Swallowed topical corticosteroids reduce the risk for long-lasting bolus impactions in eosinophilic esophagitis. Allergy 2014; 69: 1248-54.

30. Aceves SS, Bastian JF, Newbury RO, Dohil R. Oral viscous budesonide: a potential new therapy for eosinophilic esophagitis in children. Am J Gastroenterol 2007; 102: 2271-9.

31. Rawla P, Sunkara T, Thandra KC, Gaduputi V. Efficacy and safety of budesonide in the treatment of eosinophilic esophagitis: updated systematic review and meta-analysis of randomized and non-randomized studies. Drugs R D 2018; 18: 259-69.

32. Dellon ES, Sheikh A, Speck O, et al. Viscous topical is more effective than nebulized steroid therapy for patients with eosinophilic esophagitis. Gastroenterology 2012; 143: 321-4e321.

33. Schroeder S, Fleischer DM, Masterson JC, et al. Successful treatment of eosinophilic esophagitis with ciclesonide. J Allergy Clin Immunol 2012; 129: 1419-21. 
34. Moawad FJ, Veerappan GR, Dias JA, et al. Randomized controlled trial comparing aerosolized swallowed fluticasone to esomeprazole for esophageal eosinophilia. Am J Gastroenterol 2013; 108: 366-72.

35. Lipka S, Keshishian J, Boyce HW, et al. The natural history of steroid-naive eosinophilic esophagitis in adults treated with endoscopic dilation and proton pump inhibitor therapy over a mean duration of nearly 14 years. Gastrointest Endosc 2014; 80: 592-8.

36. Chuang MY, Chinnaratha MA, Hancock DG, et al. Topical steroid therapy for the treatment of eosinophilic esophagitis (EoE): a systematic review and meta-analysis. Clin Transl Gastroenterol 2015; 6: e82.

37. Golekoh MC, Hornung LN, Mukkada VA, et al. Adrenal insufficiency after chronic swallowed glucocorticoid therapy for eosinophilic esophagitis. J Pediatr 2016; 170: 240-5.

38. Hübner M, Hochhaus G, Derendorf H. Comparative pharmacology, bioavailability, pharmacokinetics, and pharmacodynamics of inhaled glucocorticosteroids. Immunol Allergy Clin North Am 2005; 25: 469-88.

39. Schoepfer AM, Straumann A, Safroneeva E. Pharmacologic treatment of eosinophilic esophagitis: an update. Gastrointest Endosc Clin N Am 2018; 28: 77-88.

40. Ko E, Chehade M. Biological therapies for eosinophilic esophagitis: where do we stand? Clin Rev Allergy Immunol 2018; 55: 205-16.

41. Bohm ME, Richter JE. Review article: oesophageal dilation in adults with eosinophilic oesophagitis. Aliment Pharmacol Ther 2011; 33: 748-57.

42. Dellon ES, Gibbs WB, Rubinas TC, et al. Esophageal dilation in eosinophilic esophagitis: safety and predictors of clinical response and complications. Gastrointest Endosc 2010; 71: 706-12.

43. Lucendo AJ, De Rezende LC, Jiménez-Contreras S, et al. Montelukast was inefficient in maintaining steroid-induced remission in adult eosinophilic esophagitis. Dig Dis Sci 2011; 56: 3551-8.

44. Straumann A, Bussmann C, Conus S, et al. Anti-TNF-alpha (infliximab) therapy for severe adult eosinophilic esophagitis. J Allergy Clin Immunol 2008; 122: 425-7.

45. Straumann A, Hoesli S, Bussmann C, et al. Anti-eosinophil activity and clinical efficacy of the CRTH2 antagonist OC000459 in eosinophilic esophagitis. Allergy 2013; 68: 375-85.

46. Stein ML, Villanueva JM, Buckmeier BK, et al. Anti-IL-5 (mepolizumab) therapy reduces eosinophil activation ex vivo and increases IL-5 and IL-5 receptor levels. J Allergy Clin Immunol 2008; 121: 1473-83.

47. Spergel JM, Rothenberg ME, Collins MH, et al. Reslizumab in children and adolescents with eosinophilic esophagitis: results of a double-blind, randomized, placebo-controlled trial. J Allergy Clin Immunol 2012; 129: 456-63.

48. Loizou D, Enav B, Komlodi-Pasztor E, et al. A pilot study of omalizumab in eosinophilic esophagitis. PLoS One 2015; 10: e0113483.

Received: 22.03.2019

Accepted: 27.03.2019 